\title{
Could MPDL3280A offer a therapeutic breakthrough in metastatic bladder cancer?
}

D espite its rising incidence and a vast amount of clinical interest, few options are available for patients diagnosed with metastatic urothelial bladder cancer (UBC), with chemotherapy remaining the standard of care. However, first-line chemotherapy is associated with a wide range of adverse effects and is poorly tolerated in a substantial number of patients, especially those who are elderly. Thus, there remains an urgent clinical need for new first-line treatments for a disease that has seen little in the way of therapeutic progress for three decades.

New data published in Nature now describe an engineered human anti-PD-L1 antibody that can be used for systemic cancer immunotherapy and that raises the possibility of a breakthrough in the treatment of metastatic UBC.

Programmed death-ligand 1 (PD-L1) is expressed on activated T cells, with expression upregulated in a number of tumour types. PD-L1 increases the propensity of cancer cells to elude immune surveillance and eradication; thus, preventing PD-L1 from interacting with its receptor could offer a means to deplete cancer cells.

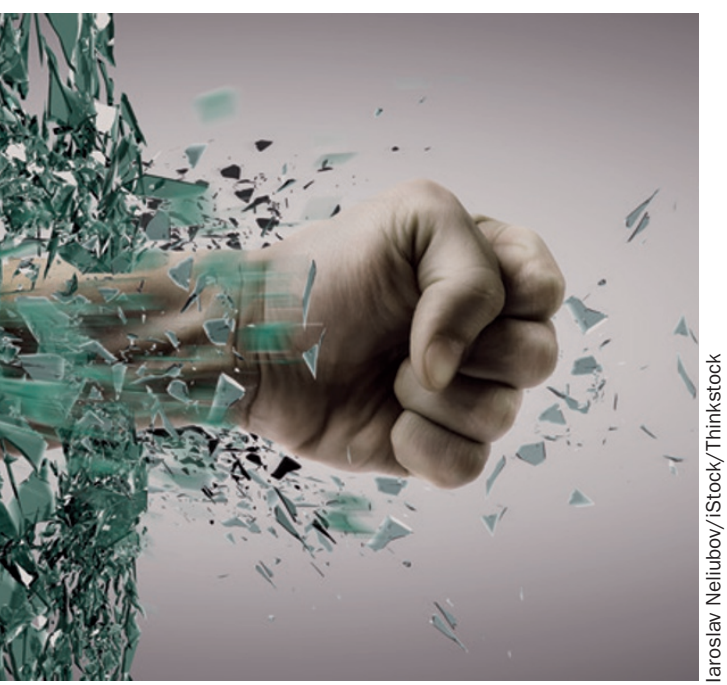

In this phase I expansion study, a multinational team examined the effect of the anti-PD-L1 antibody MPDL3280A in patients with metastatic UBC. The adaptive study design enabled the use of biomarker-enriched cohorts, with the cohort originally selected using PD-L1 immunohistochemistry (IHC) on tumour-infiltrating immune cells to identify PD-L1-positive patients. The cohort was expanded to include patients who were PD-L1-negative, in order to determine whether these patients would also respond to the antibody. IHC identified 12 patients (18\%) with tumours scored as PD-L1 IHC 0, 23 (34\%) as IHC 1, 20 (30\%) as IHC 2, 10 (15\%) as IHC 3, and 2 (3\%) as unknown, based on tumour-infiltrating immune cells. One patient had a PD-L1 IHC score of 2 or 3 for both tumour-infiltrating immune cells and tumour cells. Overall, 68 patients with UBC previously treated with chemotherapy received MPDL3280A treatment. Many of the cohort had poor prognoses, with visceral metastases $(n=50$, $75 \%) \mathrm{Hb}<10 \mathrm{~g} / \mathrm{dl}(n=12,19 \%)$, creatinine clearance $<60 \mathrm{ml} / \mathrm{min}(n=22,33 \%)$ Eastern Cooperative Oncology Group Score of 1 $(n=39,59 \%)$ or less than 3 months since their previous chemotherapy ( $n=26,42 \%)$.

Patients with UBC received a median of 65 days of MPDL3280A therapy, with $57 \%$ of them reporting adverse effects ( $4 \%$ grade 3 , no grade 4 or 5), though many were mild (grade 1 or 2 ) and transient. The objective response rates (ORRs) for patients with at least 6 weeks of follow-up data showed responses in $43 \%$ of patients (13 of 30; 95\% confidence interval [CI] 26-63\%) with IHC 2 or 3 tumours and $11 \%$ ( 4 of $35 ; 95 \%$ CI $4-26 \%$ ) of those with IHC 0 or 1 tumours. An ORR of $52 \%$ was observed in patients with IHC 2 or 3 status and at least 12 weeks of follow-up data, and 16 of the 17 patients who responded were continuing with MPDL3280A treatment at the data cut-off point.
The response duration ranged from $0.1+$ to $30.3+$ weeks for patients with IHC 2 or 3 tumours and from $0.1+$ to $6.0+$ weeks for patients with IHC 0 or 1 tumours. Response to MPDL3280A treatment was associated with IHC scores of tumour-infiltrating immune cells $(P=0.026)$, but not with those of the tumour cells $(P=0.93)$. Overall, $55 \%$ of patients demonstrated a reduction in tumour burden as measured by Response Evaluation Criteria in Solid Tumours (RECIST), and most responses were rapid, occurring at a median of just 42 days from treatment initiation.

\section{These data prompted the} FDA to grant MPDL3280A breakthrough status for UBC... 77

These data, previously reported by Nature Reviews Urology when they were presented at the ASCO conference in Chicago earlier this year, represent strong evidence that MPDL3280A treatment can achieve high response rates, and that the likelihood of response can be predicted by identifying the PD-L1 status of tumourinfiltrating immune cells. The safety profile exhibited by MPDL3280A is also encouraging, and suggests that it could be a good treatment option for older patients, who might refuse classic chemotherapy treatments owing to their associated adverse effects. These data prompted the FDA to grant MPDL3280A breakthrough status for UBC, and clinical studies to investigate MPDL3280A in bladder and other cancers are currently enrolling.

Annette Fenner

Original article Powles, T. et al. MPDL3280A (anti-PD-L1) treatment leads to clinical activity in metastatic bladder cancer. Nature 515, 558-562 (2014)

Further reading [No authors listed] From ASCO-bladder cancer: promising results for immunotherapy drug. Nat. Rev. Urol. 11, 364 (2014) 